\section{Heme as an initial treatment for severe decompensation in tyrosinemia type 1}

\section{INTRODUCTION}

When reviewing treatment guidelines published in 2017 by Chinsky et al. ${ }^{1}$ in Genetics in Medicine, following the presentation of a patient with a porphyric attack due to cessation of his nitisinone regimen, we noticed an omission for a possible life-saving treatment with heme arginate (Normosang ${ }^{\circledR}$ ).

Life-threatening acute attacks have become rare for hereditary tyrosinemia type 1 patients (HT1, MIM 276700) following the introduction of nitisinone (2-[2-nitro-4-trifluoro-methylbenzyol]-1,3 cyclohexanedione, $\operatorname{Orfadin}^{\circledast}$ ) therapy in 1992, and the application of neonatal screening. Unfortunately, interrupting nitisinone treatment can still result in the occurrence of severe neurological crises that closely resemble acute attacks seen in the acute porphyrias due to the similar rise in neurotoxic $\delta$ aminolevulinic acid (ALA). ${ }^{2}$

Therefore, to add to the review by Chinsky et al. ${ }^{1}$ we describe this HT1 patient presenting with several acute porphyric attacks (neurological crises) due to noncompliance to his nitisinone treatment. A severe attack that included neurological symptoms, and in which it was impossible to restart nitisinone orally due to nausea, was quickly resolved by immediate heme therapy.

\section{MATERIALS AND METHODS}

Clinical data of the newly presented patient were collected at the Sophia Children's Hospital, University Medical Center Rotterdam. The medical ethics committee in the Erasmus MC, Rotterdam, the Netherlands, approved the collection of retrospective/prospective data of patients with rare metabolic diseases (PRISM Biobank, MEC-2011-525). All procedures performed were in accordance with the ethical standards of the institutional research committee and with the 1964 Helsinki declaration and its later amendments. The patient provided written informed consent for the use of his data under Erasmus MC ethical committee reference number MEC-2011-525.

\section{RESULTS}

The patient was admitted three times during puberty with (porphyria-like) attacks of abdominal pain, hyponatremia, and increased plasma ALA levels.
The first episode was triggered due to increase in weight from physical inactivity; consequently, his nitisinone dosage had to be adjusted to his body weight. The second episode was due to cessation of nitisinone by the patient and was resolved by quickly restarting the patient's oral nitisinone regimen. The third episode was also due to noncompliance; however, this time the oral route was compromised due to nausea and vomiting. Also in contrast to the previous two episodes, the patient exhibited paresthesias. A markedly elevated plasma ALA confirmed the diagnosis of an acute porphyric attack, and because of the neurological involvement, combined with the compromised oral route, we started treatment with heme infusions (Normosang, $3 \mathrm{mg} / \mathrm{kg}$ ) for three days.

Laboratory tests returned to normal and vomiting stopped after three days; the abdominal pain and paresthesias subsided over the next week and he was discharged free of symptoms on the 11th day. The patient has been free of symptoms after readhering to his treatment schedule.

An illustrative timeline and relevant laboratory test results are presented in Figure S1, and in Table S1.

\section{DISCUSSION}

The rapid effect of heme infusion in this HT1 patient demonstrates its value for neurological crises or acute porphyric attacks that can be invoked by noncompliance to nitisinone. In our patient, ALA levels quickly returned to asymptomatic levels within one week after heme therapy on top of reinitiation of nitisinone. This fast recovery is similar to the recovery of inherited acute hepatic porphyric attacks, where early treatment of attacks has also been shown to promote a rapid (symptomatic) recovery. ${ }^{3}$

A benefit of heme therapy for neurological crises or acute porphyria attacks is the direct ALA lowering effect. Nitisinone indirectly lowers ALA by lowering the production and accumulation of succinylacetone (SA), and reducing SA levels leads to recovery of ALAD enzyme activity. Also nitisinone treatment is limited to the oral/ enteral route and administration is likely delayed as HT1induced porphyric attacks often present with nausea, vomiting, and constipation. ${ }^{4}$

Prior to the introduction of nitisinone in 1992, a single case report demonstrated that the acute neurological crisis of HT1 can respond successfully to heme treatment. A 21-month-old boy presented with quadriplegia since five days. Five days of hemin infusions resulted in recovery within three weeks. Following recovery he underwent liver transplantation. ${ }^{5} \mathrm{~A}$ second child was treated with heme at the age of 19 months, after she had already been quadriplegic for 13 months. A twoweek period of daily heme therapy did not improve her 
neurologic symptoms. Following liver transplantation she slowly recovered in months. ${ }^{6}$

Although morbidity and mortality from these acute porphyria-like attacks have become rare in regions with high socioeconomic standards since nitisinone was introduced, ${ }^{7}$ a Turkish cohort reported that temporary interruption of nitisinone was common for various reasons (e.g., health insurance problems $)^{8}$ and led to neurological crises in three patients and death in one patient. Also, a report from 2015 states three Iranian patients developed respiratory distress after interruption of nitisinone; two improved with full recovery two months after nitisinone therapy.

As the prognosis of acute porphyric attacks improved greatly when heme preparations became available for patients with inherited acute hepatic porphyrias, ${ }^{3}$ the observations from our case, and earlier case reports, suggest that neurological damage such as paresis, respiratory failure, and even death can be prevented when nitisinone treatment is combined or preceded by heme therapy. Nevertheless, it was not mentioned as a treatment option for acute neurological crises in recently published guidelines. ${ }^{1,9,10}$

\section{Conclusion}

We suggest that heme therapy can be life-saving when it is initiated as soon as possible during neurological crises in HT1 patients, either when nitisinone is not available as a therapy, or when the administration of nitisinone is not possible (with a restricted enteral route), or when the attacks are triggered by noncompliance. Clinicians should determine ALA levels prior to administering heme to confirm an acquired acute porphyric attack. In addition, normal ALA levels exclude an acute porphyric attack as a cause of the neurological symptoms.

\section{SUPPLEMENTARY INFORMATION}

The online version of this article (https://doi.org/10.1038/s41436$019-0658-z)$ contains supplementary material, which is available to authorized users.

\section{ACKNOWLEDGEMENTS}

We thank Edith C.H. Friesema and Rita H. Koole-Lesuis for their input and comments on the supplementary material containing biochemical data. No funding was secured for this study. R.A.N.'s position is supported by a grant from the SLO Foundation for Liver and Gastrointestinal Research.

\section{DISCLOSURE}

The authors declare no conflicts of interest.

Publisher's note Springer Nature remains neutral with regard to jurisdictional claims in published maps and institutional affiliations.

Rochus A. Neeleman, MSc ${ }^{1}$, J. H. Paul Wilson, MD (D) ${ }^{1}$, Monique Williams, $M D, P h D^{1,2}$ and

Janneke G. Langendonk, MD, PhD (D) ${ }^{1}$

${ }^{1}$ Porphyria Center Rotterdam, Center for Lysosomal and Metabolic Disorders, Department of Internal Medicine, Erasmus MC, University Medical Center Rotterdam, Rotterdam, The Netherlands; ${ }^{2}$ Department of Pediatrics, Center for Lysosomal and Metabolic Disorders, Erasmus MC-Sophia Children's Hospital, Rotterdam, The Netherlands. Correspondence: Janneke G. Langendonk (j.langendonk@erasmusmc.nl)

\section{REFERENCES}

1. Chinsky JM, Singh R, Ficicioglu $C$, et al. Diagnosis and treatment of tyrosinemia type I: a US and Canadian consensus group review and recommendations. Genet Med. 2017;19:1380.

2. Mungan NÖ, Yildizdaş D, Kör D, et al. Tyrosinemia type 1 and irreversible neurologic crisis after one month discontinuation of nitisone. Metab Brain Dis. 2016:31:1181-1183.

3. Mustajoki P, Nordmann Y. Early administration of heme arginate for acute porphyric attacks. Arch Intern Med. 1993;153:2004-2008.

4. Hall MG, Wilks MF, Provan WM, Eksborg S, Lumholtz B. Pharmacokinetics and pharmacodynamics of NTBC (2-(2-nitro-4fluoromethylbenzoyl)-1,3-cyclohexanedione) and mesotrione, inhibitors of 4-hydroxyphenyl pyruvate dioxygenase (HPPD) following a single dose to healthy male volunteers. Br J Clin Pharmacol. 2001;52:169-177.

5. Rank JM, Pascual-Leone A, Payne W, et al. Hematin therapy for the neurologic crisis of tyrosinemia. J Pediatr. 1991;118:136-139.

6. Noble-Jamieson G, Jamieson N, Clayton P, Bailey S, Ryalls M, Barnes ND. Neurological crisis in hereditary tyrosinaemia and complete reversal after liver transplantation. Arch Dis Child. 1994;70:544-545.

7. Larochelle J, Alvarez F, Bussières J-F, et al. Effect of nitisinone (NTBC) treatment on the clinical course of hepatorenal tyrosinemia in Québec. Mol Genet Metab. 2012;107:49-54.

8. Aktuglu Zeybek AC, Kiykim E, Soyucen E, et al. Hereditary tyrosinemia type 1 in Turkey: twenty year single-center experience. Pediatr Int. 2015;57:281-289.

9. Geppert J, Stinton C, Freeman K, et al. Evaluation of pre-symptomatic nitisinone treatment on long-term outcomes in tyrosinemia type 1 patients: a systematic review. Orphanet J Rare Dis. 2017;12:154-154.

10. de Laet C, Dionisi-Vici C, Leonard JV, et al. Recommendations for the management of tyrosinaemia type 1. Orphanet J Rare Dis. 2013;8:8.

Advance online publication 1 October 2019. doi:10.1038/s41436-019-0658-z 